\title{
Inclusive Charmed-Meson Production at the CERN LHC
}

\author{
B. A. Kniehl ${ }^{1}$, G. Kramer ${ }^{1}$, I. Schienbein ${ }^{2}$ and H. Spiesberger ${ }^{3}$ \\ ${ }^{1}$ II. Institut für Theoretische Physik, Universität Hamburg, \\ Luruper Chaussee 149, D-22761 Hamburg, Germany \\ ${ }^{2}$ Laboratoire de Physique Subatomique et de Cosmologie, \\ Université Joseph Fourier Grenoble 1, \\ CNRS/IN2P3, Institut National Polytechnique de Grenoble, \\ 53 avenue des Martyrs, F-38026 Grenoble, France \\ ${ }^{3}$ Institut für Physik, Johannes-Gutenberg-Universität, \\ Staudinger Weg 7, D-55099 Mainz, Germany
}

\begin{abstract}
We present predictions for the inclusive production of charmed hadrons at the CERN LHC in the general-mass variable-flavor-number scheme at next-to-leading order. Detailed numerical results are compared to data where available, or presented in a way to ease future comparisons with experimental results. We also point out that measurements at large rapidity have the potential to pin down models of intrinsic charm.
\end{abstract}




\section{Introduction}

Past measurements of charmed-hadron, $X_{c}$, production in high-energy scattering experiments [1] have shown that inclusive heavy-quark production provides us with an interesting testing ground for the dynamics of the strong interaction. With the advent of high-statistics data from the LHC, we can expect that upcoming new results will allow us to test the predictions of QCD with much better precision. The LHC experiments will also extend into the region of higher transverse momenta, where theory is expected to provide more reliable predictions. This is not only important for testing QCD itself; but a good understanding of heavy-quark production is also vital for searches of new-physics phenomena.

Recent analyses of $D$-meson production at the LHC have been published by the ALICE [2] and ATLAS collaborations [3]. Similar data that are being analyzed by the LHCb collaboration [4] are expected to appear soon. The aim of the present article is to present predictions for the inclusive production of charmed hadrons at the LHC within the generalmass variable-flavor-number scheme (GM-VFNS). We extend results which have already been used by the experimental collaborations for comparisons with their data, and we discuss these results under a common perspective. Where possible we, therefore, use information from the experiments about the accessible phase space regions. In a recent paper [5], we have considered the inclusive production of $B$ mesons, for which experimental results from the CMS collaboration are already available [6, 7, 8].

Theoretical predictions for the production of heavy-flavored mesons at high transverse momentum $\left(p_{T}\right)$ are technically difficult to obtain due to the presence of two different scales, $p_{T}$ and the heavy-quark mass $m$. On the one hand, the heavy-quark mass can be considered as the large scale, since $m>\Lambda_{\mathrm{QCD}}$, making perturbative QCD applicable. When $m$ is the only large scale, as for example in the calculation of the total cross section or in predictions for $p_{T}$ distributions at small values of $p_{T}$, i.e. if $p_{T}$ is of the same order of magnitude as $m$, predictions from a fixed-order calculation are reliable. On the other hand, if the transverse momentum of the produced heavy quark is large compared with the heavy-quark mass, $p_{T} \gg m$, then large logarithms $\ln \left(p_{T}^{2} / m^{2}\right)$ have to be resummed.

In the first case, for $p_{T} \lesssim m$, the traditional approach is called fixed-flavor-number scheme (FFNS) [9]. It is based on the assumption that the gluon and the light partons $(u, d, s)$ are the only active partons. The heavy quark appears only in the final state, produced in the hard-scattering process of light partons. The heavy-quark mass is explicitly taken into account together with the transverse momentum of the produced heavy meson assuming that $m$ and $p_{T}$ are of the same order. Predictions of the FFNS are reliable close to the threshold of heavy-quark production.

In the second case, for $p_{T} \gg m$, the large logarithmic terms have to be resummed. The well-known factorization theorem provides the foundation of this resummation by incorporating the large logarithms into parton distribution and fragmentation functions and imposing the DGLAP evolution equations. This approach requires that the heavy 
quark is treated as a parton, and as a consequence, one has to take into account additional processes where heavy quarks occur as incoming partons or by fragmentation from light partons. The heavy quark is treated as any other massless parton. If $m$ is neglected in the calculation of the hard-scattering matrix element, this approach is called zero-mass variable-flavor-number scheme (ZM-VFNS) [10]. The predictions in the ZM-VFNS are expected to be reliable only in the region of very large transverse momenta, since terms of the order of $m^{2} / p_{T}^{2}$ present in the hard-scattering cross sections are neglected.

In fact, it is not necessary to neglect $m$ altogether in a variable-flavor-number scheme. Instead, it is possible to absorb the large logarithms $\ln \left(p_{T} / m\right)$ into parton distribution and fragmentation functions, where they are resummed by imposing DGLAP evolution, as in the ZM-VFNS, while at the same time, $m$-dependent terms as obtained in the FFNS are retained in the hard-scattering cross sections. This so-called general-mass variable-flavornumber scheme (GM-VFNS) thus combines the virtues of the FFNS and the ZM-VFNS. The required subtraction of logarithmic terms, which are related to initial- and final-state singularities, can be defined using the usual $\overline{\mathrm{MS}}$ prescription, which guarantees the universality of parton distribution and fragmentation functions and allows for a meaningful comparison of data from different measurements. This approach has been applied in the calculation of charmed-meson production in $p \bar{p}$ collisions in Ref. [11] and compared to data from the CDF collaboration [1] at the Fermilab Tevatron.

\section{Setup and Input}

The theoretical background and explicit analytic results of the GM-VFNS approach have been discussed in detail previously in Ref. [11] and the references cited therein. Here we only describe our choice of input for the present numerical analysis.

Throughout we use as parton distribution functions (PDFs) the set CTEQ6.6 [12] as implemented in LHAPDF [13], except where we discuss uncertainties related to the choice of the PDFs. The fragmentation functions (FFs) determined in Ref. [14] are used wherever possible, i.e. for the production of $D^{0}, D^{ \pm}$, and $D^{* \pm}$ mesons. They are based on fits to the presently most precise data on charmed-meson production from the CLEO collaboration [15] at LEPP CESR and from the Belle collaboration [16] at the KEK collider for $B$ physics (KEKB). These FFs always refer to the average over charge-conjugated states, and our results below have to be understood as averaged cross sections $(\sigma(D)+\sigma(\bar{D})) / 2$. For $D_{s}$ and $\Lambda_{c}$ production, we have to resort to the earlier determination of FFs described in Ref. [17]. These FFs were determined by fitting the fractional-energy spectra of the $X_{c}$ hadrons measured by the OPAL collaboration [18, 19] in $e^{+} e^{-}$annihilation on the $Z$ boson resonance at the CERN LEP1 collider. These data have rather large experimental errors and the disadvantage of being at the rather large scale of the $Z$-boson mass, far away from the typical scales of $X_{c}$ production presently observed at the LHC.

The subtractions related to renormalization as well as to the factorization of initial- and 
final-state singularities requires the introduction of scale parameters $\mu_{R}, \mu_{I}$, and $\mu_{F}$. We fix these scales by the transverse mass of the produced charm quark, $m_{T}=\sqrt{p_{T}^{2}+m^{2}}$. In order to exploit the freedom in the choice of scales, we introduce scale parameters $\xi_{i}$ $(i=R, I, F)$ by setting $\mu_{i}=\xi_{i} m_{T}$. Error bands describing the theoretical uncertainties can then be determined by varying the values of $\xi_{i}$ independently by factors of two up and down while keeping any ratio of the $\xi_{i}$ smaller than or equal to two.

We emphasize that the uncertainties related to scale variations are by far the dominating source of theoretical uncertainties. For PDF-related uncertainties, we will show explicit results below. For the influence of variations of the value of the charm-quark mass we refer to our previous work [11. There we have shown that those terms that carry a dependence on $m$, coming from the hard-scattering matrix element, are small. Therefore, we always keep $m=1.5 \mathrm{GeV}$ in the present work.

\section{Numerical Results}

The ALICE collaboration has published results for the differential cross section $d \sigma / d p_{T}$ at $\sqrt{s}=7 \mathrm{TeV}$ in the central rapidity range $|y| \leq 0.5$. In Fig. 1 , we show the predictions of the GM-VFNS for $p_{T} \geq 3 \mathrm{GeV}$, where we identify $y$ with the pseudorapidity of the produced heavy meson. We include a comparison with the data points of ALICE taken from Ref. 2] for the production of $\left(D^{0}+\bar{D}^{0}\right) / 2,\left(D^{+}+D^{-}\right) / 2$, and $\left(D^{*+}+D^{*-}\right) / 2$. An error band due to scale variations as described in the preceding section is also shown. The agreement between theory and experiment is good except at the largest values of $p_{T}$, where the data lie somewhat below the error band. Data for the smaller center-of-mass energy $\sqrt{s}=2.76 \mathrm{TeV}$, also for $|y| \leq 0.5$, are currently under investigation. We, therefore, present results for this forthcoming analysis in Fig. 2 using the same conventions as in Fig. 1 .

At low transverse momentum, $p_{T} \lesssim 3 \mathrm{GeV}$, scale uncertainties are large and amount to about $\pm 65 \%$; they decrease to values below $+30 \%(-15 \%)$ at $p_{T} \simeq 30 \mathrm{GeV}$. In Fig. 3 a, we show the cross section ratios for $d \sigma / d p_{T}$ normalized to the GM-VFNS default prediction with $\xi_{i}=1$. Also the data points of the ALICE experiment are normalized to the GM-VFNS result with $\xi_{i}=1$. At $p_{T} \lesssim 3$, the data are much smaller than the default prediction. However, it turns out that a very good description of the data also in the first three $p_{T}$ bins can be obtained by choosing $\xi_{I}=\xi_{F}=0.7$ (see the full histogram). This choice of scales corresponds to setting $\mu_{i}=\sqrt{m_{T}^{2} / 2}$.

We repeat that the uncertainties of theoretical predictions due to scale variations are dominating. This can be seen by comparing the results shown in Fig. 3a with those in Fig. $3 \mathrm{~b}$, where we present ratios of $d \sigma / d p_{T}$ for different choices of the PDF parametrizations with $\xi_{I}=\xi_{F}=0.7$. All results including the data are normalized to the default evaluation using the PDF set CTEQ6.6. We notice that all PDFs reproduce the data very well inside the experimental errors except in the first $p_{T}$ bin, where the default prediction, 
corresponding to unit ratio, lies outside the error. All other PDF choices, i.e. MSTW08NLO [20], NNPDF2.1 [21], HERPDF1.5-NLO [22], and CT10 [23], yield predictions inside the experimental errors.

We should emphasize here that, with our choice of scale parameters, $\mu_{I}$ and $\mu_{F}$ can fall below $m$ at the lowest $p_{T}$ values. In our implementation, we freeze the scales at
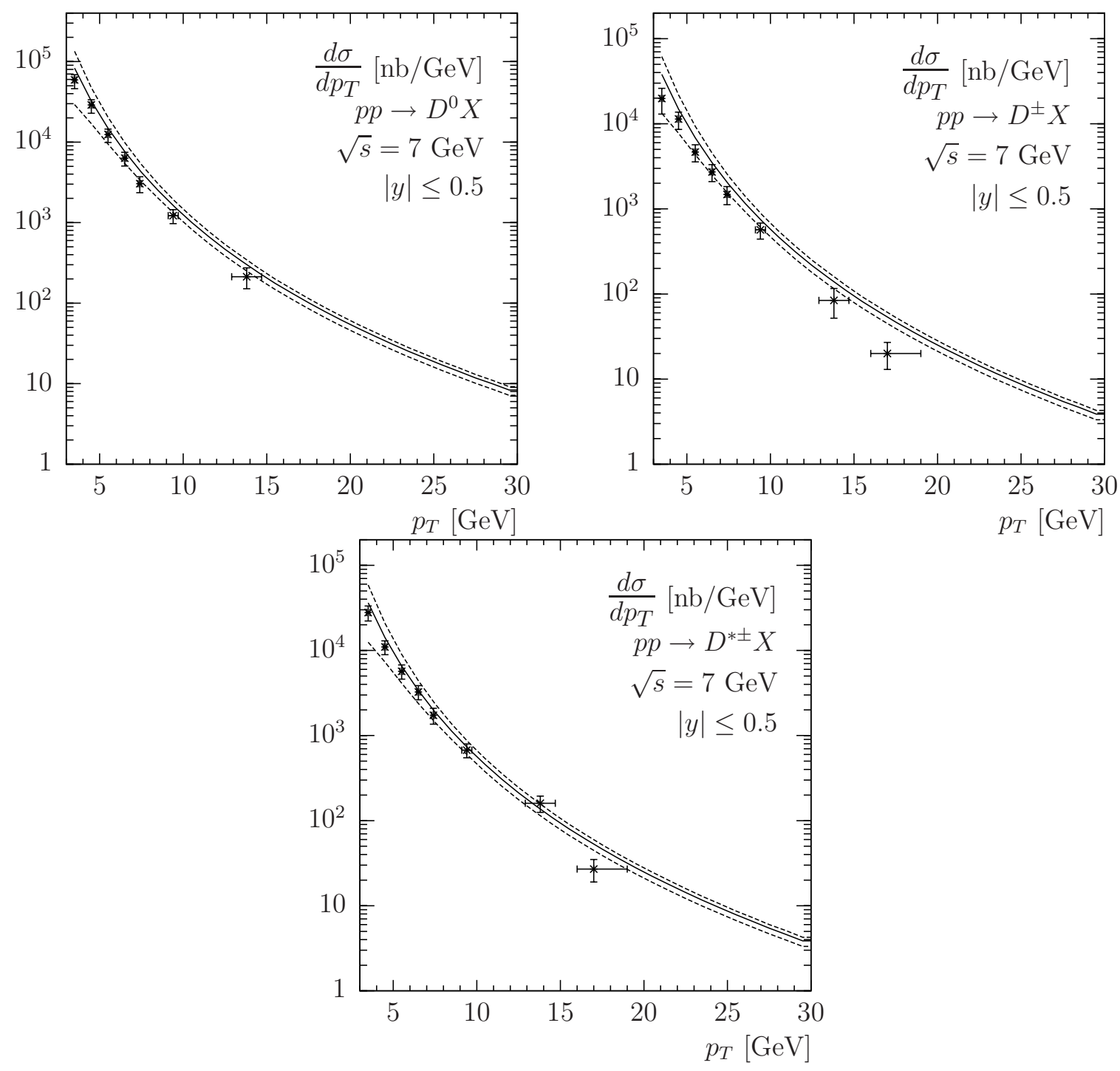

Figure 1: Differential cross section $\mathrm{d} \sigma / \mathrm{d} p_{T}$ as a function of $p_{T}$ for $p+p \rightarrow D+X$ with (a) $D=D^{0}$, (b) $D=D^{ \pm}$, and (c) $D=D^{* \pm}$ integrated over rapidity in the range $-0.5 \leq y \leq 0.5$ for $\sqrt{s}=7 \mathrm{TeV}$ at NLO in the GM-VFNS using the FFs of Ref. [14] and the CTEQ6.6 PDFs. The full lines are obtained for the default choice of scale parameters $\xi_{R}=\xi_{I}=\xi_{F}=1$, and the error band (dashed lines) are from independent variations of $\xi_{R}, \xi_{I}$, and $\xi_{F}$ as described in the text. The data points are taken from Ref. [2]. 
$\mu_{I}=\mu_{F}=m=1.5 \mathrm{GeV}$, so that the PDFs and FFs do not evaluate to zero. The observed strong suppression in the lowest $p_{T}$ bin in Fig. 3b is then partly due to the fact that the value of $m$ used in the more recent PDF fits is $m=1.4 \mathrm{GeV}$, and not $m=1.3$ $\mathrm{GeV}$ as in the case of CTEQ6.6. As a consequence, the charm PDF of set CTEQ6.6 is larger due to the longer evolution path as compared to MSTW08-NLO, NNPDF2.1, and HERAPDF1.5-NLO.

Actually, due to the different values of $m$ used in the PDF fits, there is some residual $m$ dependence of the predicted cross sections at low values of $p_{T}$. The value $m=1.5 \mathrm{GeV}$ used in our calculation agrees with the one in the FF fits from Ref. [14], but not with

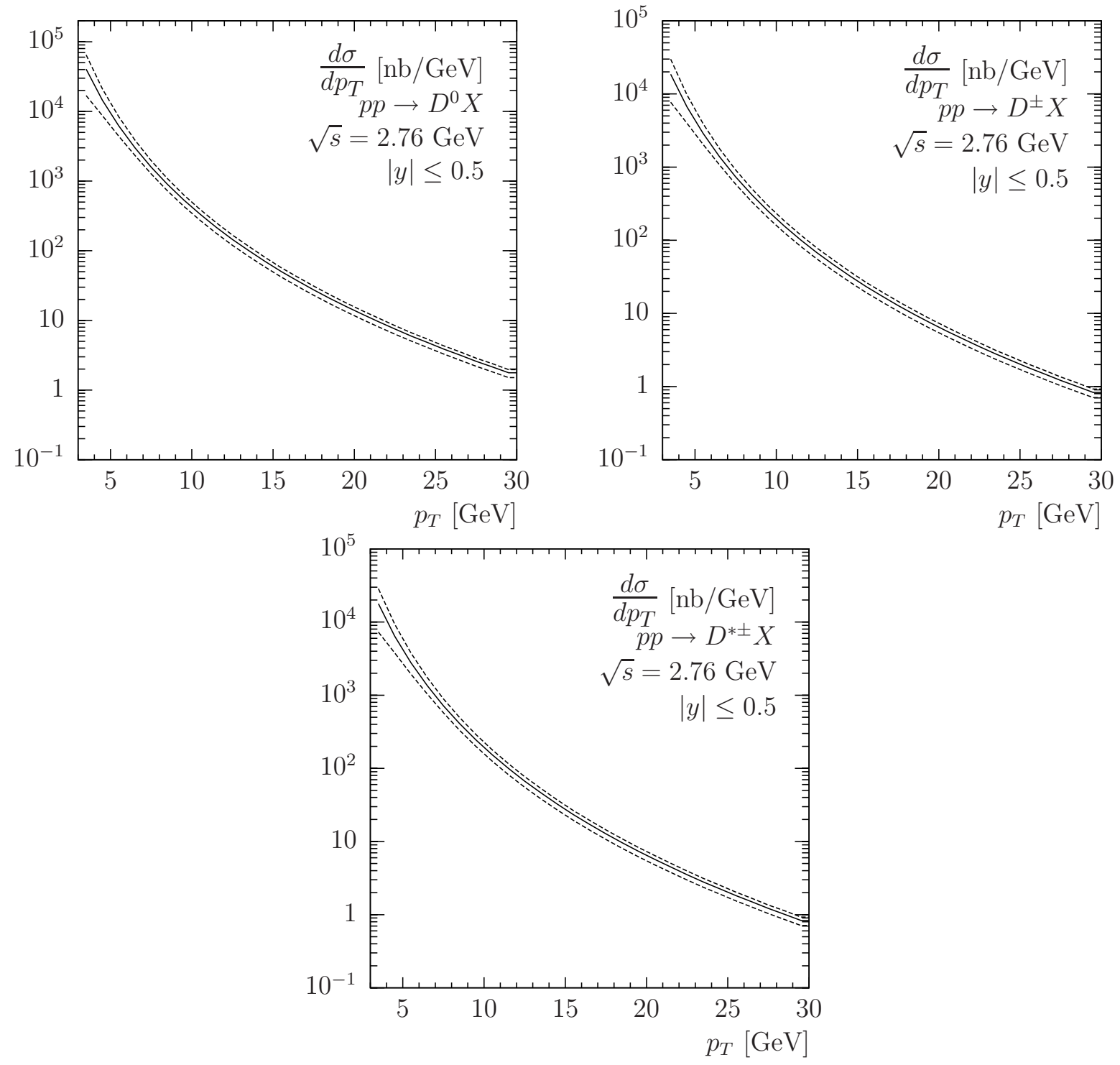

Figure 2: Same as Fig. 1 but for $\sqrt{s}=2.76 \mathrm{TeV}$. 
the one in the PDFs used here. For example, CTEQ6.6 and CT10 use $m=1.3 \mathrm{GeV}$ while in the parametrizations MSTW08-NLO, NNPDF 2.1, and HERAPDF 1.5 (NLO) $m=1.4 \mathrm{GeV}$ is adopted. For consistency, one should use the same value of $m$ in all three components of the cross section formula, PDFs, FFs and hard-scattering matrix elements. This would, however, require separate fits of the FFs as functions of $m$, a task which is left for future studies. Similarly, it would not completely be consistent to use different values of $\alpha_{s}$ in the partonic cross sections and in the PDFs and FFs. For our default choice of PDFs, CTEQ6.6, as well as for CT10, our choice $\alpha_{s}\left(M_{Z}^{2}\right)=0.118$ agrees with what was used in the PDF and FF fits. For MSTW08-NLO and NNPDF 2.1, the value of $\alpha_{s}$ is only slightly larger than for CTEQ6.6, and for HERAPDF 1.5 (NLO) the value $\alpha_{s}\left(M_{Z}^{2}\right)=0.1176$ was used, which is nearly the same as for CTEQ6.6. We are thus confident that our evaluations are consistent with respect to the choice of $\alpha_{s}$.

Although the GM-VFNS calculation is able to describe the data at the lowest values of $p_{T}$ if the factorization scales are fixed by choosing $\xi_{I}=\xi_{F}=0.7$, one cannot expect a fully satisfactory description for $p_{T} \rightarrow 0$. This is due to contributions with a charm quark in the initial state, where a massless approach is required. In the low- $p_{T}$ range,

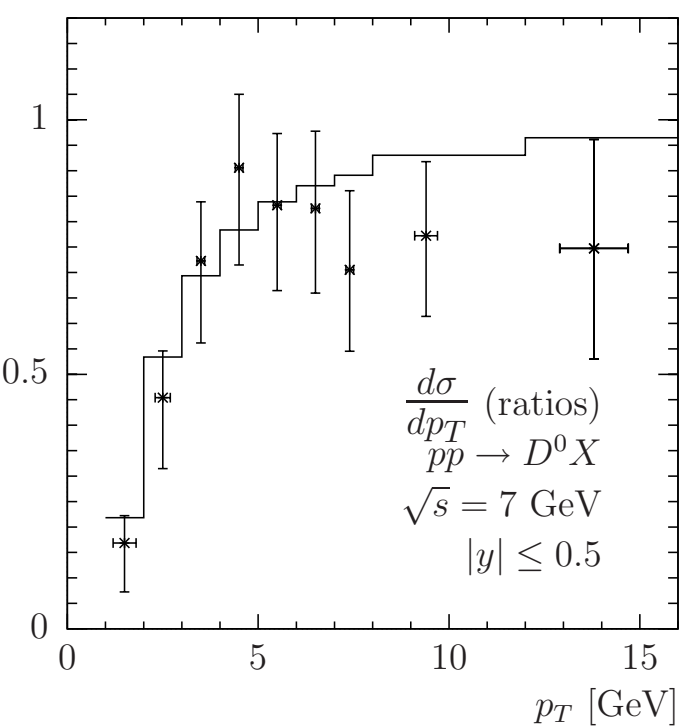

(a)

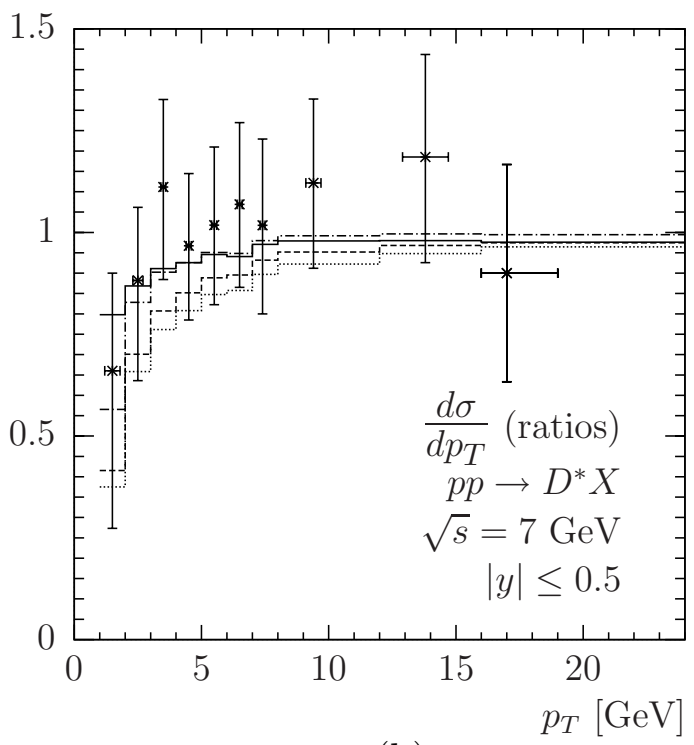

(b)

Figure 3: (a) Ratios of $d \sigma / d p_{T}$ for $D^{0}$-meson production at ALICE at $\sqrt{s}=7 \mathrm{TeV}$. All cross sections are normalized to the GM-VFNS prediction with $\xi_{i}=1$. Data points, also normalized to the GM-VFNS calculation, are taken from Ref. [2]. The histogram is obtained using $\xi_{I}=\xi_{F}=0.7$ and $\xi_{R}=1$. (b) Ratios of $d \sigma / d p_{T}$ for $D^{*}$-meson production at ALICE at $\sqrt{s}=7 \mathrm{TeV}$ for different PDFs. All cross sections are normalized to the GMVFNS prediction with $\xi_{I}=\xi_{F}=0.7$. Data points are taken from Ref. [2] and normalized to the GM-VFNS calculation with $\xi_{I}=\xi_{F}=0.7$. Full histogram: CT10 [23], dashed: MSTW08-NLO [20], dotted: NNPDF 2.1 [21], dash-dotted: HERAPDF 1.5 (NLO) [22]. 
one expects the FFNS approach to be more reliable. A comparison of the predictions of the GM-VFNS and the FFNS for the case of $D^{0}$ production, using $\xi_{i}=1$, is shown in Fig. 4. The FFNS calculation is done without including a FF; the transition from the charm quark to the charmed meson is, however, taken into account by multiplying the parton-level results with the branching ratio $B R\left(c \rightarrow D^{0}\right)=0.62$. One can observe that the FFNS describes the turn-over towards the production threshold at low values of $p_{T}$ correctly, but it fails to describe the data in the high- $p_{T}$ tail. This demonstrates the importance of resumming terms involving the large collinear logarithm, $\ln \left(p_{T} / m\right)$, which is done in the GM-VFNS, but not in the FFNS. The resummation is also responsible for the considerable reduction of the size of the error band from scale variations. Taking into account a scale-independent FF may improve the agreement between the FFNS and the data at high values of $p_{T}$, but the theory loses its predictability here, since the FF can not be defined in a universal way in the FFNS. From Fig. 4, one can also read off that the curves from the GM-VFNS and the FFNS meet each other at about $p_{T} \simeq 5 \mathrm{GeV}$. This would be the $p_{T}$-value where the theoretical prescription should switch from a fixed to a variable-flavor-number scheme. We do not follow this possibility here, but leave a study of the matching between the GM-VFNS and the FFNS to future work.

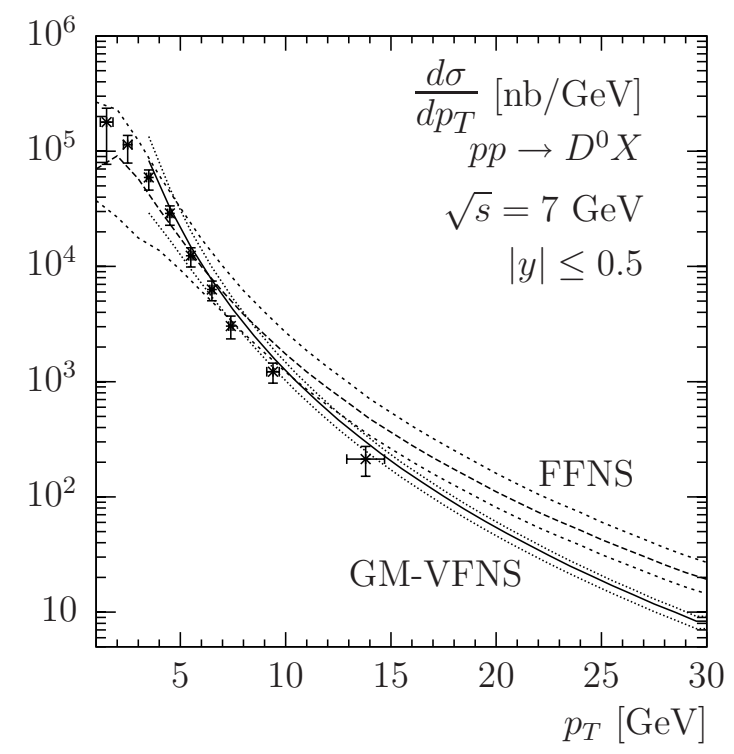

Figure 4: $p_{T}$ distribution $\mathrm{d} \sigma / \mathrm{d} p_{T}$ for $p+p \rightarrow D^{0}+X$ integrated over rapidity in the range $|y| \leq 0.5$ for $\sqrt{s}=7.0 \mathrm{TeV}$ at NLO in the GM-VFNS (full line) as in Fig. 1, compared with the results of the FFNS (dashed line). Dotted lines describe the corresponding error bands from scale variations as described in the text. The points with error bars are data from the ALICE collaboration [2]. 
Now we present results for a comparison with experimental data from the ATLAS collaboration following the analysis described in Ref. [3]. The corresponding measurement covers a larger $p_{T}$ range, up to $p_{T}=40 \mathrm{GeV}$; also the $y$ range is wider and extends up to $y=2.1$. We choose the bins in $p_{T}$ and $y$ as in Ref. [3]. Figures 5 and 6 show results for $d \sigma / d p_{T}$ and $d \sigma / d y$, respectively, for $D^{0}, D^{ \pm}$, and $D^{*}$-meson production. Again, these results are for the average of the charge-conjugated states. We also remark that an experimentally relevant contribution coming from the fragmentation of $b$ quarks into $D$ mesons is not taken into account in our theoretical predictions. The behavior of these differential cross
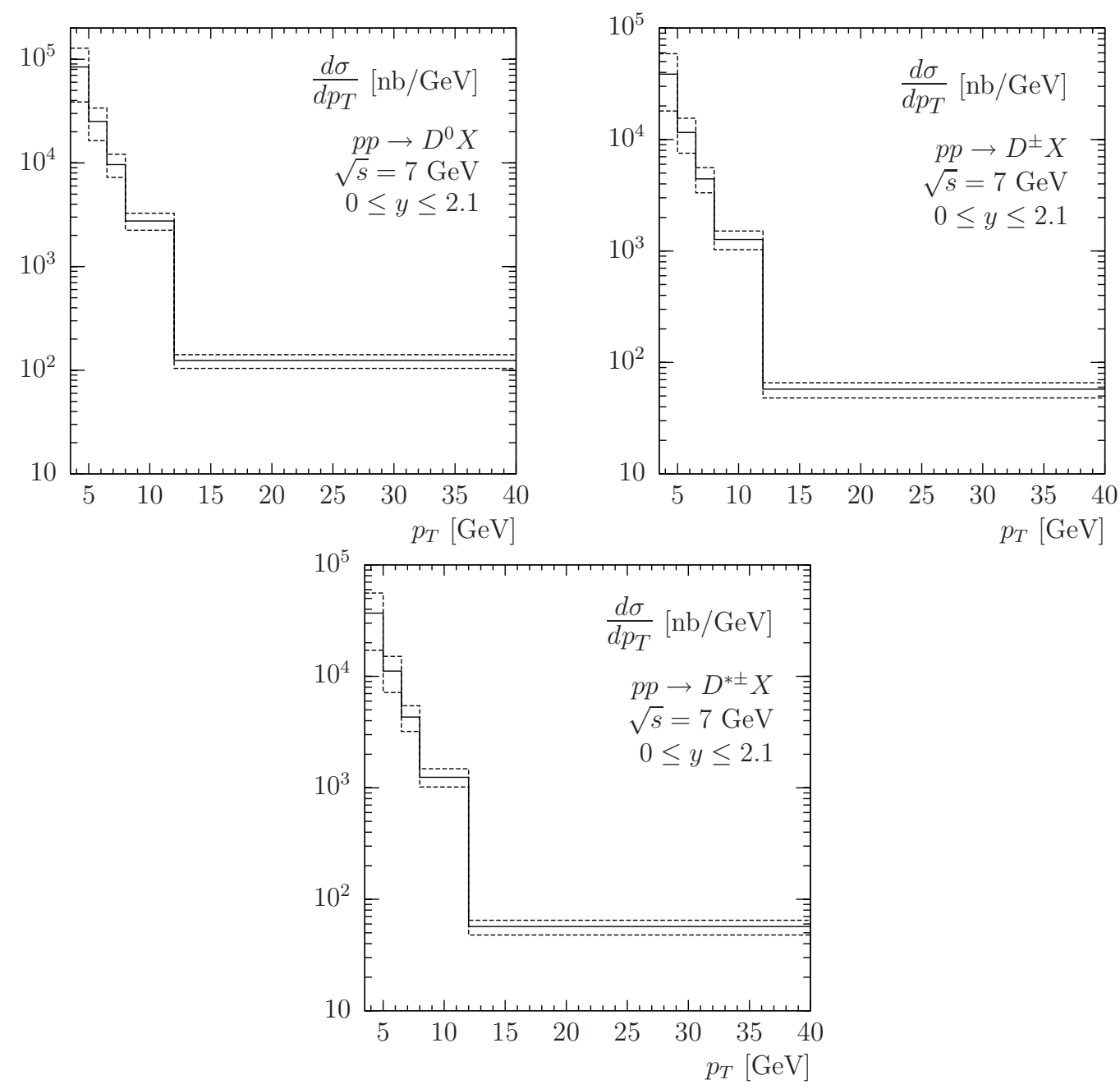

Figure 5: $\quad p_{T}$ distributions $\mathrm{d} \sigma / \mathrm{d} p_{T}$ of $p+p \rightarrow D+X$ with (a) $D=D^{0}$, (b) $D=D^{ \pm}$, and (c) $D=D^{* \pm}$ integrated over rapidity in the range $0 \leq y \leq 2.1$ for $\sqrt{s}=7.0 \mathrm{TeV}$ at NLO in the GM-VFNS using the FFs of Ref. [14] and the CTEQ6.6 PDFs. The full histograms are obtained for the default choice of scale parameters, $\xi_{R}=\xi_{I}=\xi_{F}=1$, and the error bands (dashed histograms) are from independent variations of $\xi_{R}, \xi_{I}$, and $\xi_{F}$ as described in the text. 
sections is similar to what we found above for the case of the ALICE data, i.e. we observe large scale variations at low values of $p_{T}$. The comparison with the ATLAS data as shown in Ref. [3] reveals that our calculations are in good agreement with the data within the observed large uncertainties.
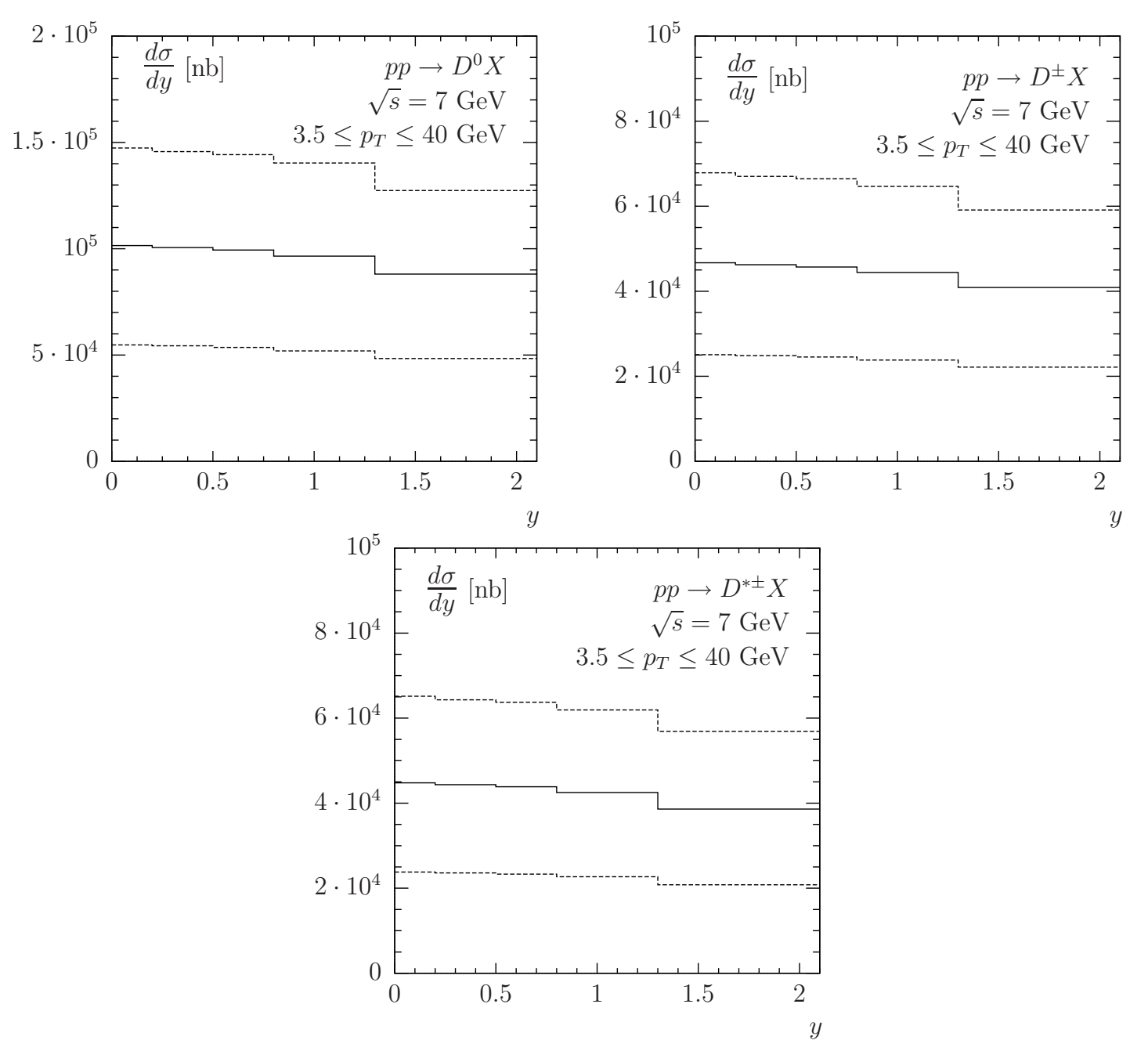

Figure 6: Rapidity distributions $\mathrm{d} \sigma / \mathrm{d} y$ of $p+p \rightarrow D+X$ with (a) $D=D^{0}$, (b) $D=D^{ \pm}$, and (c) $D=D^{* \pm}$ integrated over transverse momentum in the range $3.5 \mathrm{GeV} \leq p_{T} \leq 40$ $\mathrm{GeV}$ for $\sqrt{s}=7.0 \mathrm{TeV}$ at NLO in the GM-VFNS using the FFs of Ref. [14. The full histograms are obtained for the default choice of scale parameters, $\xi_{R}=\xi_{I}=\xi_{F}=1$, and the error bands (dashed histograms) are from independent variations of $\xi_{R}, \xi_{I}$, and $\xi_{F}$ as described in the text. 


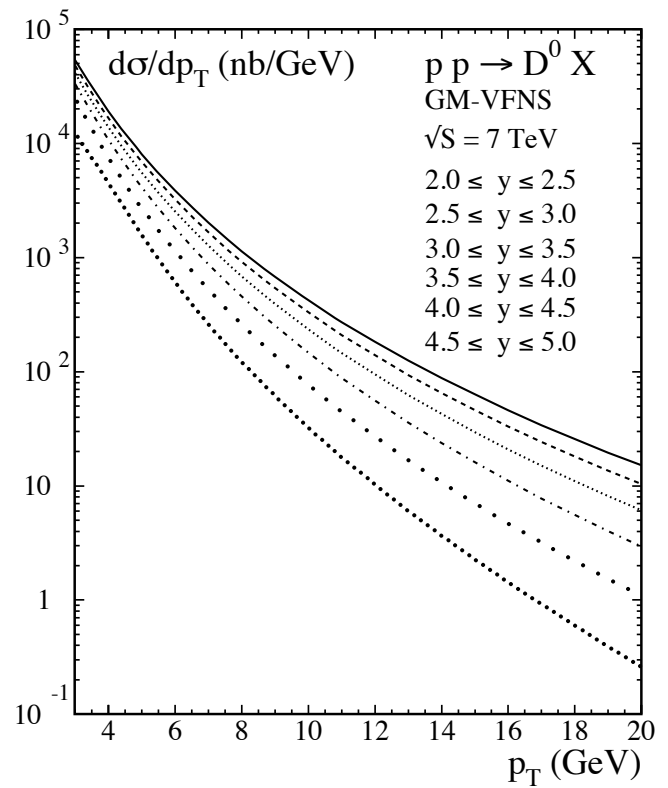

(a)

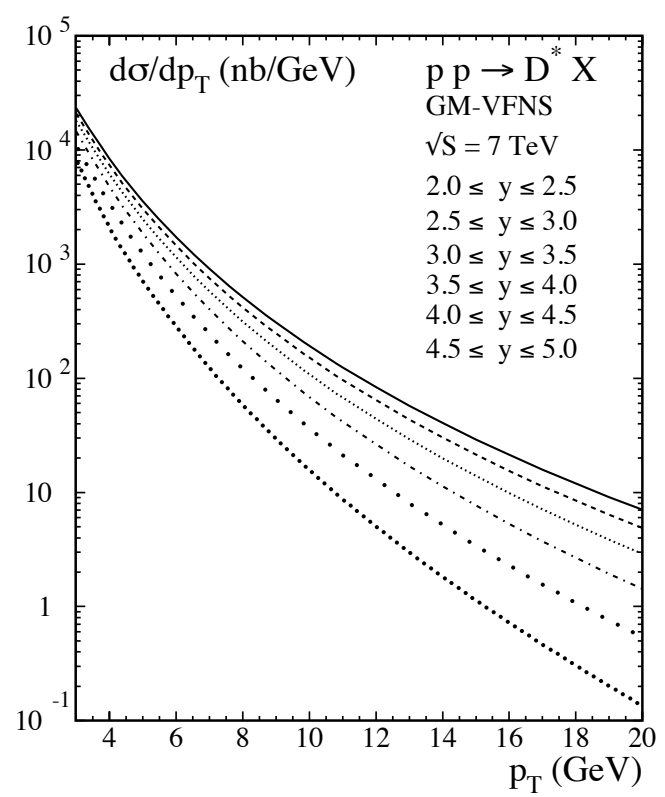

(c)

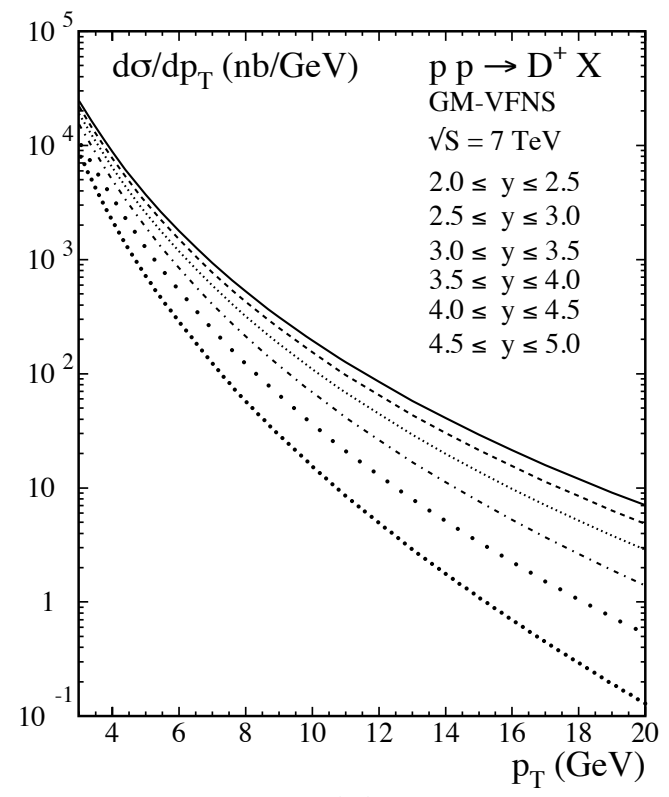

(b)

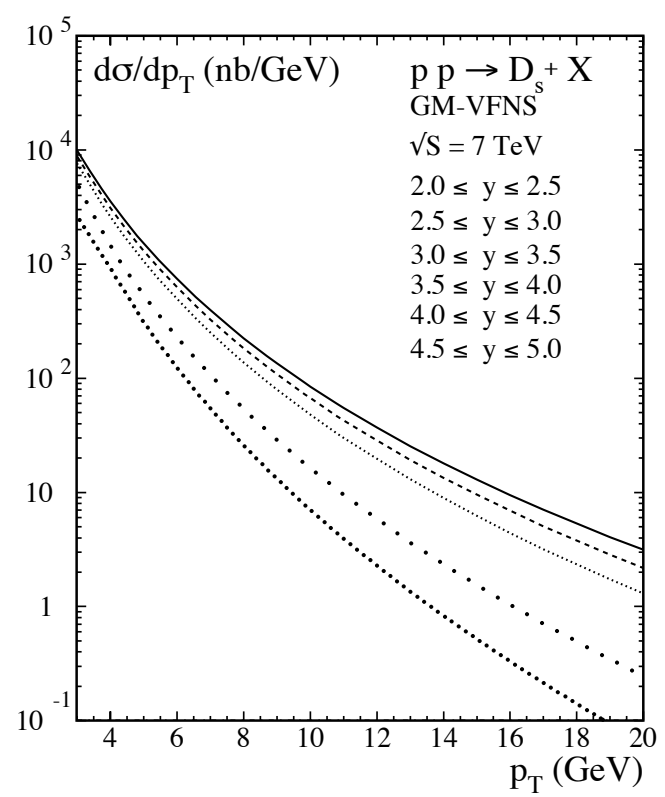

(d)

Figure 7: $\quad p_{T}$ distributions $\mathrm{d} \sigma / \mathrm{d} p_{T}$ of $p+p \rightarrow D+X$ with (a) $D=D^{0}$, (b) $D=D^{ \pm}$, (c) $D=D^{* \pm}$, and (d) $D=D_{s}^{ \pm}$for $\sqrt{s}=7 \mathrm{TeV}$ at NLO in the GM-VFNS using the FFs of Ref. [14 for $D^{0}, D^{ \pm}$, and $D^{* \pm}$ and the FFs of Ref. [17] for $D_{s}^{ \pm}$. The various lines represent the default predictions for $\xi_{R}=\xi_{I}=\xi_{F}=1$, integrated over the rapidity regions indicated in the figures (larger rapidities correspond to smaller cross sections). 
The cross sections are largest at central rapidities and fall off towards larger values of $|y|$. This is shown in Fig. 7 for $D^{0}, D^{+}, D^{*}$ and $D_{s}$ mesons and in Fig. 8 for $\Lambda_{c}$ baryons. Here we present results for the $p_{T}$ distributions in six $|y|$ bins of width $\Delta y=0.5$ between $y=2.0$ and $y=5.0$. Corresponding measurements made by the LHCb collaboration have been presented at conferences [4], and a publication is expected soon. At $p_{T} \simeq 3 \mathrm{GeV}$, the cross section goes down with increasing rapidity by a factor of 3 to 5 , depending on the type of the produced hadron; at $p_{T} \simeq 20 \mathrm{GeV}$, the decrease with rapidity amounts to almost a factor of 50. Again, uncertainties due to scale variations are large at small values of $p_{T}$ and decrease towards larger values of $p_{T}$. The corresponding results including also the theory error bands are shown in Fig. 9 for the case of $D^{0}$-meson production in the various rapidity bins. Within these errors, there is agreement with data as shown in Ref. [4].

At large rapidities, it is interesting to study the influence of nonperturbative contributions to the charm-quark content of the incoming proton, usually called intrinsic charm. An enhancement of the charm PDF $c\left(x, \mu_{F}\right)$ at $x>0.1$ can be visible in the cross section at large rapidities. The CTEQ collaboration has implemented appropriate models, which are compatible with the global data samples, in their PDF parametrization CTEQ6.5 [24]. In

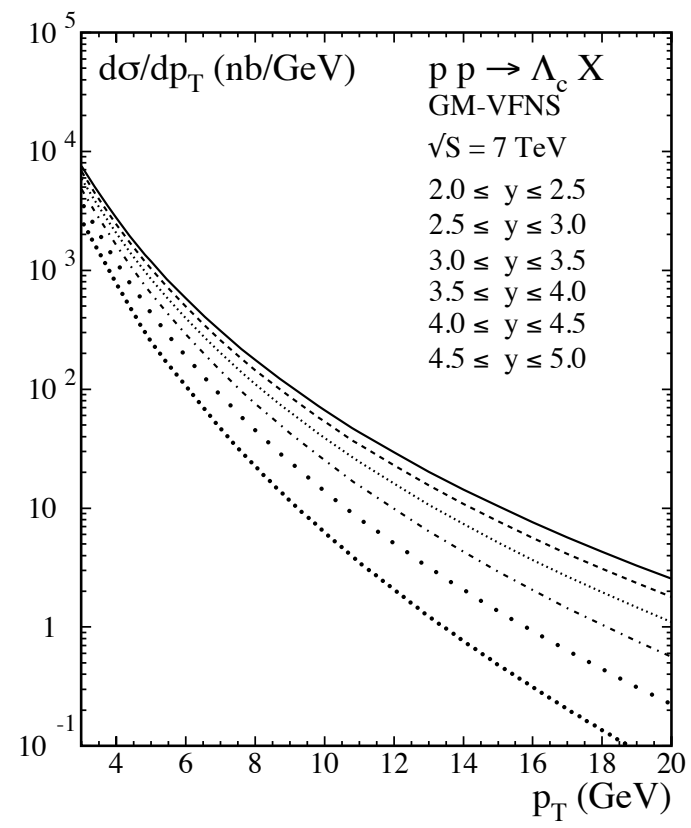

Figure 8: $\quad p_{T}$ distributions $\mathrm{d} \sigma / \mathrm{d} p_{T}$ of $p+p \rightarrow \Lambda_{c}+X$ for $\sqrt{s}=7 \mathrm{TeV}$ at NLO in the GM-VFNS using the FFs of Ref. [17]. The various lines represent the default predictions for $\xi_{R}=\xi_{I}=\xi_{F}=1$, integrated over the rapidity regions indicated in the figure (larger rapidities correspond to smaller cross sections). 

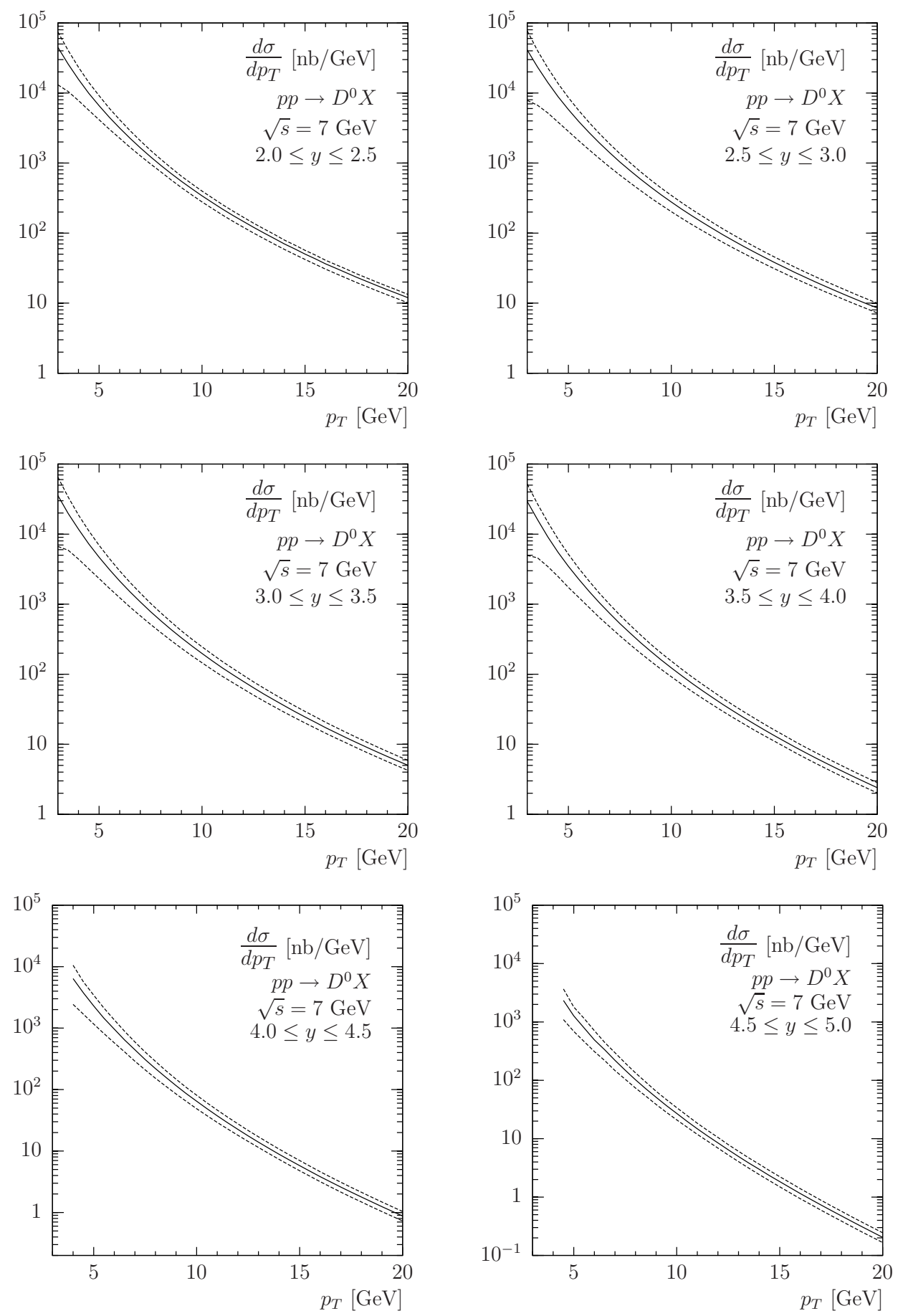

Figure 9: $p_{T}$ distributions $\mathrm{d} \sigma / \mathrm{d} p_{T}$ of $p+p \rightarrow D^{0}+X$ at $\sqrt{s}=7 \mathrm{TeV}$ in different rapidity bins as indicated in the figures. We show the uncertainty bands from scale variations following the prescription described in the text. The FFs are taken from Ref. [14]. 
our recent work [25], we studied the impact of these models on possible measurements at the Tevatron and at BNL RHIC. Here, we use the more recent parametrization CTEQ6.6 [12] and show corresponding results for the relative enhancements of the $p_{T}$ distributions in bins of rapidity in Fig. 10. We have selected two models (see Ref. [24] for details): Fig. 10a shows the calculation using the BHPS model with a $3.5 \%(c+\bar{c})$ content in the proton (at the scale $\mu_{F}=1.3 \mathrm{GeV}$ ), Fig. 10b refers to the model of a high-strength sea-like charm component. We show results for $D^{0}$ production, but the cross section ratios for other $D$ mesons are very similar. One observes large enhancements, increasing with rapidity, and in the first model also with $p_{T}$. These numerical results show that it should be possible to exclude or narrow down models for intrinsic charm with forthcoming data from the $\mathrm{LHCb}$ experiment.

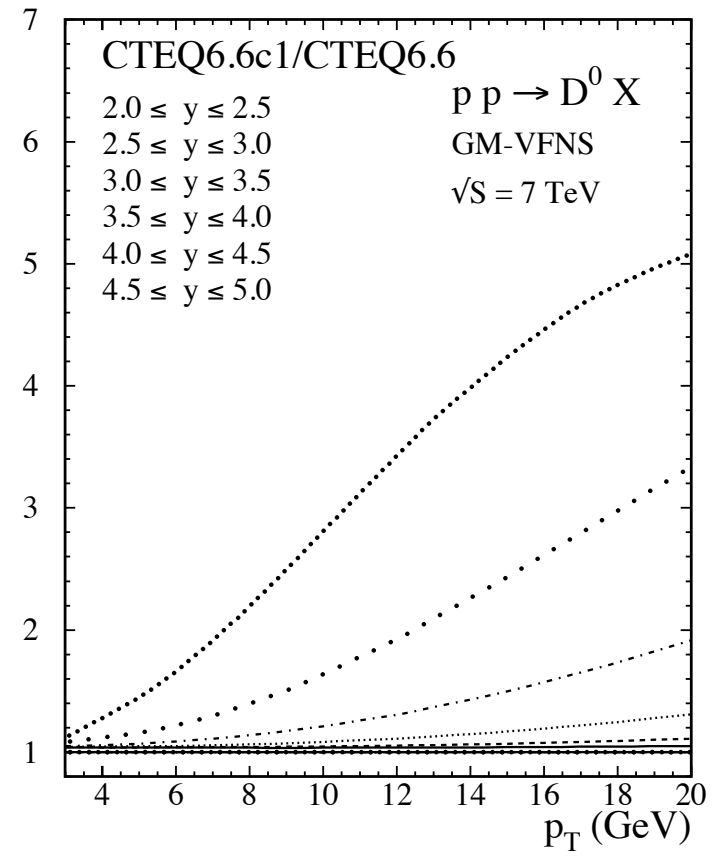

(a)

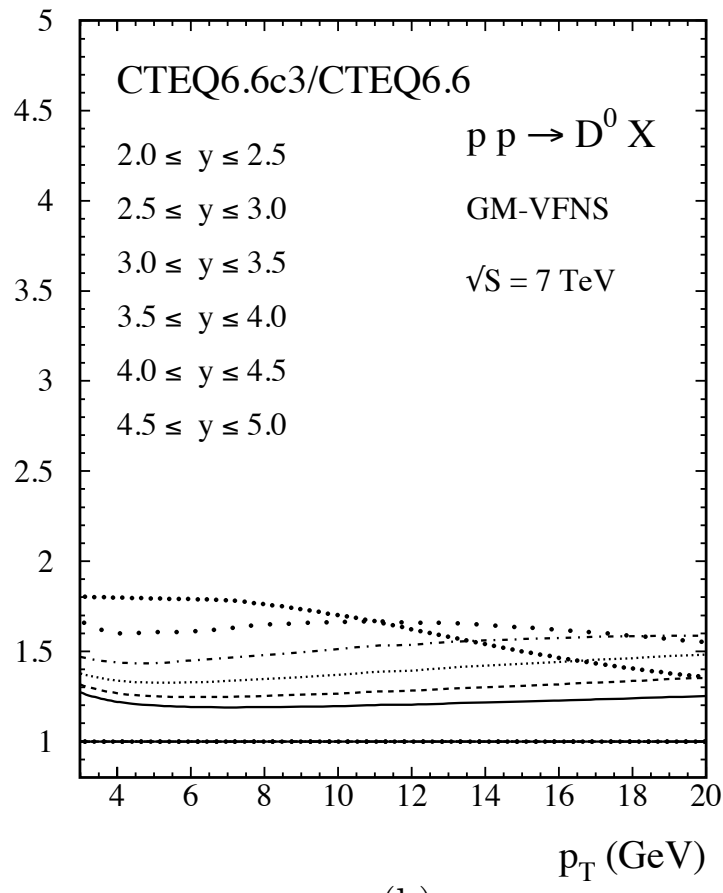

(b)

Figure 10: Ratio of the $p_{T}$ distributions $\mathrm{d} \sigma / \mathrm{d} p_{T}$ for $p+p \rightarrow D^{0}+X$ at NLO in the GM-VFNS, using different models of intrinsic charm: (a) BHPS model with $3.5 \%(c+\bar{c})$ content (at $\mu_{F}=1.3 \mathrm{GeV}$ ), (b) model with a high-strength sea-like charm component. The FFs are taken from Ref. [14] and $\sqrt{s}=7 \mathrm{TeV}$. The various lines represent the default predictions for $\xi_{R}=\xi_{I}=\xi_{F}=1$, integrated over the rapidity regions indicated in the figures (larger rapidities correspond to larger cross section ratios everywhere in (a) and at small $p_{T}$ in (b)). 


\section{Conclusions}

In summary, we applied the GM-VFNS to obtain NLO predictions for the production of charmed mesons in $p p$ collisions at the LHC. Experimental data from the ALICE collaboration are already published, and agreement with our predictions is in general good, even at low values of $p_{T}$ if the factorization scale parameters are chosen appropriately. We expect more data from the other LHC experiments soon, and our results have been presented in a form which should make future comparisons straightforward.

We have found that the production cross sections at large rapidities are sensitive to a nonperturbative component of the charm parton distribution function. Measurements should soon be able to exclude models which are still allowed by previous data.

\section{Acknowledgments}

We thank our experimental colleagues from the LHC collaborations A. Dainese, P. Thompson, L. Gladilin, and M. Schmelling for discussions about the experimental results.

This work was supported in part by the German Federal Ministry for Education and Research BMBF through Grant No. 05 HT6GUA, by the German Research Foundation DFG through Grant No. KN 365/7-1, and by the Helmholtz Association HGF through Grant No. Ha 101.

\section{References}

[1] D. Acosta et al. (CDF Collaboration), Phys. Rev. Lett. 91, 241804 (2003) hepex/0307080.

[2] B. Abelev et al. (ALICE Collaboration), arXiv:1111.1553 [hep-ex].

[3] The ATLAS Collaboration, ATL-PHYS-PUB-2011-012; ATLAS-CONF-2011-017.

[4] The LHCb Collaboration, LHCb-CONF-2010-013. To be published in 6th Int. Workshop on the CKM Unitarity Triangle, Warwick, UK, 6-10 Sep. 2010.

[5] B. A. Kniehl, G. Kramer, I. Schienbein, and H. Spiesberger, Phys. Rev. D 84, 094026 (2011) arXiv:1109.2472 [hep-ph]].

[6] V. Khachatryan et al. (CMS Collaboration), Phys. Rev. Lett. 106, 112001 (2011) arXiv:1101.0131 [hep-ex]].

[7] S. Chatrchyan et al. (CMS Collaboration), Phys. Rev. Lett. 106, 252001 (2011) arXiv:1104.2892 [hep-ex]]. 
[8] S. Chatrchyan et al. (CMS Collaboration), Phys. Rev. D 84, 052008 (2011) arXiv:1106.4048 [hep-ex]].

[9] P. Nason, S. Dawson, and R. K. Ellis, Nucl. Phys. B 303, 607 (1988); B 327, 49 (1989); B 335, 260(E) (1989); W. Beenakker, H. Kuijf, W. L. van Neerven, and J. Smith, Phys. Rev. D 40, 54 (1989); W. Beenakker, W. L. van Neerven, R. Meng, G. A. Schuler, and J. Smith, Nucl. Phys. B 351, 507 (1991); S. Frixione, M. Mangano, P. Nason and G. Ridolfi, Phys. Lett. B 348, 633 (1995); S. Frixione, P. Nason and G. Ridolfi, Nucl. Phys. B 545, 3 (1995); I. Bojak and M. Stratmann, Phys. Rev. D 67, 034010 (2003) |arXiv:hep-ph/0112276.

[10] M. Cacciari and M. Greco, Nucl. Phys. B 421, 530 (1994) arXiv:hep-ph/9311260]; B. A. Kniehl, M. Krämer, G. Kramer, and M. Spira, Phys. Lett. B 356, 539 (1995) arXiv:hep-ph/9505410]; M. Cacciari, M. Greco, B. A. Kniehl, M. Krämer, G. Kramer, and M. Spira, Nucl. Phys. B 466, 173 (1996) |arXiv:hep-ph/9512246]; J. Binnewies, B. A. Kniehl, and G. Kramer, Z. Phys. C 76, 677 (1997) |arXiv:hepph/9702408]; B. A. Kniehl, G. Kramer, and M. Spira, Z. Phys. C 76, 689 (1997) |arXiv:hep-ph/9610267]; J. Binnewies, B. A. Kniehl, and G. Kramer, Phys. Rev. D 58, 014014 (1998) arXiv:hep-ph/9712482]; Phys. Rev. D 58, 034016 (1998) arXiv:hep-ph/9802231]; B. A. Kniehl and G. Kramer, Phys. Rev. D 60, 014006 (1999) |arXiv:hep-ph/9901348]; B. A. Kniehl, in Proceedings of the 14th Topical Conference on Hadron Collider Physics: Hadron Collider Physics 2002, Karlsruhe, Germany, 2002, edited by M. Erdmann and Th. Müller (Springer, Berlin, 2003), p. 161 [arXiv:hep-ph/0211008]; B. A. Kniehl and G. Kramer, Phys. Rev. D 71, 094013 (2005) [arXiv:hep-ph/0504058]; Phys. Rev. D 74, 037502 (2006) arXiv:hepph/0607306.

[11] B. A. Kniehl, G. Kramer, I. Schienbein, and H. Spiesberger, Phys. Rev. D 71, 014018 (2005) arXiv:hep-ph/0410289 ; Eur. Phys. J. C 41, 199 (2005) arXiv:hepph/0502194; AIP Conf. Proc. 792, 867 (2005) arXiv:hep-ph/0507068; Phys. Rev. Lett. 96, 012001 (2006) [arXiv:hep-ph/0508129]; Phys. Rev. D 79, 094009 (2009) arXiv:0901.4130 [hep-ph]].

[12] P. M. Nadolsky et al. (CTEQ Collaboration), Phys. Rev. D 78, 013004 (2008) arXiv:0802.0007 [hep-ph]].

[13] LHAPDF, the Les Houches Accord PDF Interface, URL: http://projects.hepforge.org/lhapdf/pdfsets.

[14] T. Kneesch, B. A. Kniehl, G. Kramer, and I. Schienbein, Nucl. Phys. B 799, 34 (2008) arXiv:0712.0481 [hep-ph]].

[15] M. Artuso et al. (CLEO Collaboration), Phys. Rev. D 70, 112001 (2004) arXiv:hepex/0402040. 
[16] R. Seuster et al. (Belle Collaboration), Phys. Rev. D 73, 032002 (2006) arXiv:hepex/0506068.

[17] B. A. Kniehl and G. Kramer, Phys. Rev. D 74, 037502 (2006) arXiv:hepph/0607306.

[18] G. Alexander et al. (OPAL Collaboration), Z. Phys. C 72, 1 (1996).

[19] K. Ackerstaff et al. (OPAL Collaboration), Eur. Phys. J. C 1, 439 (1998) arXiv:hepex/9708021.

[20] A. D. Martin, W. J. Stirling, R. S. Thorne, and G. Watt, Eur. Phys. J. C 63, 189 (2009) arXiv:0901.0002 [hep-ph]].

[21] R. D. Ball, V. Bertone, F. Cerutti, L. Del Debbio, S. Forte, A. Guffanti, J. I. Latorre, and J. Rojo et al., Nucl. Phys. B 849, 296 (2011) arXiv:1101.1300 [hep-ph]].

[22] H1 and ZEUS Collaborations, ZEUS-prel-10-018 and H1prelim-10-142; ZEUS-prel11-001 and H1prelim-11-034; ZEUS-prel-11-002 and H1prelim-11-042; A. MCooperSarkar, arXiv:1112.2107 [hep-ph]. To be published in: Proceedings of the 2011 Europhysics Conference on High Energy Physics-HEP-2011.

[23] H. L. Lai, M. Guzzi, J. Huston, Z. Li, P. M. Nadolsky, J. Pumplin, and C. P. Yuan, Phys. Rev. D 82, 074024 (2010) arXiv:1007.2241 [hep-ph]].

[24] J. Pumplin, H. L. Lai, and W. K. Tung, Phys. Rev. D 75, 054029 (2007) arXiv:hepph/0701220.

[25] B. A. Kniehl, G. Kramer, I. Schienbein, and H. Spiesberger, Phys. Rev. D 79, 094009 (2009) arXiv:0901.4130 [hep-ph]]. 